\title{
B-3. Biochemical Studies of Human Gliomas
}

\author{
Shozo Nakazawa, Namiko SaIto and Komei Ueki \\ Department of Neurosurgery, Brain Research Institute, Niigata University
}

Slices of surgically removed human gliomas weighing approximately $80-90 \mathrm{mg}$ were incubated in a Krebs-Ringer phosphate medium containing glucose as a substrate and the respiratory activities were measured using the standard Warburg manometric technique.

For the studies of protein biosynthesis, the slices were incubated in medium containing $\left[\mathrm{U}-{ }^{14} \mathrm{C}\right]$ leucine and proteins were isolated from the TCA insoluble fraction, weighed and counted in a gas flow counter.

The results indicate that the rate of oxygen uptake of human gliomas is about $1 / 5$ lower as compared with that of human brain tissue.

In studying the elevated $\mathrm{KCl}(105 \mathrm{mM}$ ) in the medium, the oxygen uptake is decreased by using the slices of human gliomas. On the other hand, it is increased markedly by using the slices of human cortex. However, the specific activity in protein of human gliomas is about 5-10 times higher than that of human cortex tissues.

The rate of an increase in the specific activity of protein is usually comparable with malignancy of gliomas.

It can be seen from these results that protein biosynthesis of gliomas is very active by using the energy from glycolytic process.

\section{B-4. Melatonin and HIMOT in Pinealoma}

\author{
Osamu Nakai, and Noboru Mryazawa \\ Department of Neurosurgery, Brain Research Institute Niigata University \\ Department of Neuropathology, Brain Research Institute, Niigata University
}

Extensive investigations concerning the origin of pinealoma and the relation between pineal region tumor and precocious puberty has been done. These problems, however, has still remained to be solved.

Recently, it has been evident that HIOMT, an enzyme which forms melatonin, is exclusively localized in the pineal gland and that melatonin has an inhibitory effect to the gonads. In an assumption that these observations may provide a clue in the solution of the above mentioned problems, HIOMT activity of pinealomas and the developmental evolution of HIOMT in human fetus and rats were examined.

HIOMT activity in the human pineal glands from autopsy materials, pinealomas, the rat and bovine pineal glands were measured after Axelrod and Wurtman's 
method. Mean HIOMT activity in 6 human pineal glands is $44 \mathrm{p}$ moles/hr/mg. w. w., which accords with the result of Wurtman. HIOMT activity is found neither in 2 pinealomas of two cell pattern, nor in human fetus of the 6 months gestation.

In general, the biochemical phenotype of neoplasms resembles that of normal undifferenciated cells. Thus, our results may not provide the convincing evidence that 2 pinealomas have their origins in the pineal gland.

The developmental evolution of HIOMT activity was examined in rats. The activity begins to appear 9 days after birth and increases gradually. The highest activity/body weight is found 20 days prior to the onset of pubescence.

A pineal gland, therefore, may play a possible role in induction of pubescence and sexual maturation. The hypothalamic dysfunction or the gonadotropin secretion by tumor has been emphasized as a cause of the precocious puberty in patients with pineal region tumor. However, our present study suggested that pineal gland dysfunction also should be considered to be responsible for inducing precocious puberty.

\title{
B-5. Quantitative Compositions of Fatty Acids in Brain Tumor Tissue and Gerebrospinal Fluid
}

\author{
Yoshihisa Onodera, Masamichi Hasue, \\ Yukio Yamamoto and Tetsuro Miwa \\ Department of Neurosurgery, Tokyo Medical College
}

Normal brain consists of a great deal of lipids. It, however, does not almost utilize lipids for energy.

On the other hand, tumors take fatty acids from their hosts and utilize for energy. From this point of view, we have studied the metabolism of fatty acids in brain tumors.

As material, we used tumor tissue and CSF in various brain tumors: these are 5 gliomas, 5 meningiomas and 5 metastatic carcinomas.

The samples separated from each material were analized by gaschromatography, and each fatty acid were performed by internal standard method using arachidic acid.

The quantitative error of this method was within $\pm 5 \%$.

The composition of fatty acids in tumor tissue differed from normal brain, for example, C18: 2, which is not almost contained in normal brain, appeared in tumor tissue, and the contents of fatty acids in brain tumors were lesser than these of normal brain. The various types of brain tumor, however, were generally similar qualitatively and quantitatively.

The composition of fatty acids in CSF of brain tumor were related to disturbance of consciousness, and was not affected by histological difference in tumor.

When consciousness is clear, the composition of fatty acids in CSF were similar to those of normal qualitatively, but each fatty acid decreased quantitatively. 